\title{
Crystallization of amorphous boron by the calorimetric method
}

\author{
Tengiz Machaladze ${ }^{1}$, Madona Samkharadze ${ }^{2}$, Nino Kakhidze², Maia Makhviladze ${ }^{2}$ \\ ${ }^{1}$ Rafiel Agladze Institute of Inorganic Chemistry and Electrochemistry, I. Javakhishvili Tbilisi State University, Tbilisi, Georgia \\ ${ }^{2}$ Akaki Tsereteli State University, Kutaisi, Georgia \\ Email:nino.kakhidze@gmail.com
}

Received 27 November 2013; revised 27 December 2013; accepted 4 January 2014

Copyright (C) 2014 Tengiz Machaladze et al. This is an open access article distributed under the Creative Commons Attribution License, which permits unrestricted use, distribution, and reproduction in any medium, provided the original work is properly cited. In accordance of the Creative Commons Attribution License all Copyrights (C) 2014 are reserved for SCIRP and the owner of the intellectual property Tengiz Machaladze et al. All Copyright (C) 2014 are guarded by law and by SCIRP as a guardian.

\section{ABSTRACT}

The kinetics of formation of crystal boron was studied by $\mathrm{X}$-ray structure and differential-thermal analyses. The enthalpy of transition of amorphous boron to crystal boron with formation of an intermediate non-equilibrium phase was determined by the calorimetric method. Studies were carried out by using the differential scanning calorimeter SETARAM. Amorphous boron received by diboron cracking was used as the starting material. The test sample is characterized by stability even at a high temperature. When heated, amorphous boron first transforms to crystal boron of $\alpha$-modification, and during a further heating, there occur several phase transitions, which means the transition of $\alpha$-rhombohedric crystal boron to the $\beta^{\prime}$ and $\beta^{\prime \prime}$ metastable states. Studies of various modifications were carried out by radiographic and electro-optic methods. The high sensitiveness of the calorimeter made it possible to control temperature in the oven, make records and detect even the smallest thermal effects.

\section{KEYWORDS}

Amorphous boron; $\alpha, \beta$-Rombohedric Crystalline Phase; Calorimeter; Thermogram

\section{INTRODUCTION}

Elementary boron exists in the form of various crystalline $\alpha, \beta$-rhombohedric and tetragonal modifications as well as in amorphous state. The heating of boron in vacuum or in an inert gas medium brings about its crystallization. Boron undergoes some transformations until the stable crystalline $\beta$-rhombohedric phase is formed. The transformation process depends on various factors: sample heating conditions, content of impurities and the presence of defects in the structure of the initial amorphous boron [1].

The kinetics of crystalline boron formation was studied by X-ray diffraction and differential-thermal analyses in [2-4], but thermal effect values were not determined. The $D_{\alpha} \rightarrow D_{\beta}$ transformation was studied by the electro-optical method in [5].

\section{EXPERIMENTAL}

The enthalpy of transformation of amorphous boron to the crystalline state with formation of an intermediate non-equilibrium state was measured by the calorimetric method. Experimental studies were carried out using the differential electronic scanning microcalorimeter HT1500. This high-quality calorimetric apparatus made by SETARAM company is able to control the conditions in the kiln, to record signals automatically and to measure even the minutest thermal effects about 20 micro J $(0.0005 \mathrm{cal})$.

The calorimeter was calibrated against the known metal melting heat values. Calorimetric and thermal signals were processed through the amplifier which delivered signals simultaneously to the recorder and to the numerical printing integrator which, upon the completion of transformation, gave out the corresponding numerical values. The integrator provides the high accuracy of integration since it directly processes the signal and not the signal copy, registered at another device.

The standard compounds were weighed to an accuracy of $0.0005 \mathrm{~g}$. After putting the melting pot with tested samples into the calorimeter, they were subjected to degassing. Experiments were run in the argon steam medium at the pressure $P=1.0 \times 10^{-5} \mathrm{~Pa}$.

The calorimeter constant $K$ for the melting point of the 
standard compound was determined by the formula:

$$
K=\frac{\Delta H}{A} \times \frac{G}{M},
$$

where $\Delta H$ is the melting heat of the standard compound, cal/mole; $A$ is the number of pulses produced by the generator of the amplifier for the electromotive force of the differential thermal battery which corresponds to the transformation area, in $\mathrm{mV}$; $G$ is the weight of the standard compound; $M$ is the molecular mass of the standard compound.

The standard compounds used for the calorimeter calibration were tin, lead, zinc, aluminum, potassium chloride, silver, gadolinium, silicon and iron of “c·p" qualification.

The calorimeter constant $K$ was calculated for each standard compound by averaging the values of $8-10$ measurements. The standard error was determined by the formula:

$$
\Delta S= \pm 2 \sqrt{\frac{\sum(\Delta i)^{2}}{n(n-1)}}
$$

where $n$ is the number of experiments; $\Delta i$ is a deviation from the average value.

\section{RESULT AND DISCUSSIONS}

The dependence of the calorimeter constant on temperature is shown in Figure 1.

Experiments with the investigated compounds were run similarly to experiments with the standard compounds. The phase transition heat was calculated by the formula

$$
\Delta H=K \times A \frac{M}{G},
$$

where $K$ is the calorimeter constant shown by the calibration curve, $A$ is the number of pulses produced by the generator of the amplifier for the electromotive force of the differential thermal battery, corresponds to the transformation area, in $\mathrm{mV}$; $M$ is the molecular mass of the investigated compound, $G$ is the investigated compound weight.

To determine the experiment accuracy, we studied the transformation $F_{e_{\beta}} \rightarrow F_{e_{\gamma}}$. The difference between our measurements and those given in [6] is approximately $1 \%$. Amorphous boron received by diboron cracking was used as the starting material. The test sample is characterized by stability even at a high temperature. When heated, amorphous boron first transforms to crystal boron of $\alpha$-modification, and during a further heating there occur several phase transitions, which means the transition of $\alpha$-rhombohedric crystal boron to the $\beta^{\prime}$ and $\beta^{\prime \prime}$ metastable states.

Studies of various modifications were carried out by radiographic and electro-optic methods. The high sensitiveness of the calorimeter made it possible to control temperature in the oven, make records and detect even the smallest thermal effects.

Amorphous boron selected as the initial material was prepared by cracking diboron. Its purity was 99.5\%.

No thermal effect was observed when heating the boron sample up to $1300^{\circ} \mathrm{C}$ at the rate of 200,400 , $600^{\circ} \mathrm{C}$ per hour. However, with a further increase of the heating temperature by $200^{\circ}$ per hour there occur two different exothermal peaks: the first peak is observed in the range $1360-1400^{\circ} \mathrm{C}$ (Figure 2) and the second occurs immediately upon the completion of the first peak and attains its maximum at $1450^{\circ} \mathrm{C}$.

The X-ray and electro-optical analysis confirmed the transformation of amorphous boron to crystalline boron of $\alpha$-modification. The enthalpy change during the transformation process was $\Delta H=-4.45 \mathrm{kcal} / \mathrm{mole}$. The standard error was equal to $\Delta S= \pm 1.5 \%$. A further heating of the sample revealed several phases of transformations with a maximum $1550^{\circ} \mathrm{C}, 1662^{\circ} \mathrm{C}$ and $1712^{\circ} \mathrm{C}$ (Figure 3). The X-ray study showed that as a result of these transformations $\alpha$-rhombohedral crystalline boron transforms to the metastable phase $\beta^{\prime}$ and $\beta^{\prime \prime}$. Transformation at $1712^{\circ} \mathrm{C}$ is associated with the final formation of the $\beta$-rombohedric phase. The thermal effect, correspond-

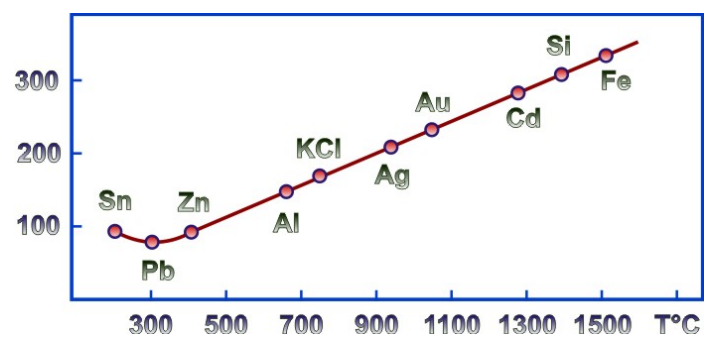

Figure 1. Calibration curve for calorimeter HT-1500.

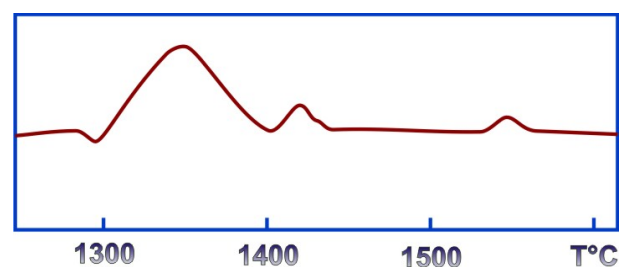

Figure 2. Thermogram of phase transformation of amorphous boron to $\alpha$-boron.

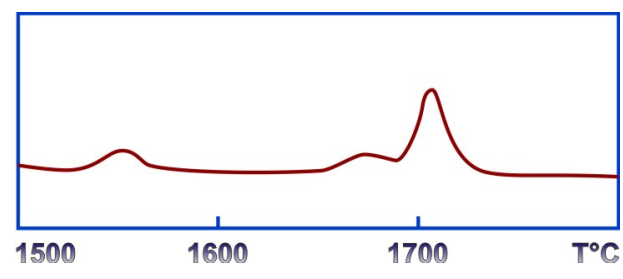

Figure 3. Thermogram of phase transformation of $\alpha$-boron to $\beta$-boron. 
ing to the variation of system enthalpy, comprises $\Delta H=-1.05 \mathrm{kcal} / \mathrm{mole}$.

\section{CONCLUSIONS}

Amorphous boron received by diboron cracking was used as the starting material. The test sample is characterized by stability even at a high temperature. When heated, amorphous boron first transforms to crystal boron of $\alpha$-modification, and during a further heating, there occur several phase transitions, which means the transition of $\alpha$-rhombohedric crystal boron to the $\beta^{\prime}$ and $\beta^{\prime \prime}$ metastable states.

Studies of various modifications were carried out by radiographic and electro-optic methods. The high sensitiveness of the calorimeter made it possible to control temperature in the oven, make records and detect even the smallest thermal effects.

\section{REFERENCES}

[1] Lavrinenko, V.A., Gogotsi, Yu.G. and Fratzevich, I.N.
(1984) High-temperature oxidation of hot pressed boron. Transactions of Academy of Sciences of USSR, 275, 114-117. (in Russian)

[2] Bagramashvili, I.A. and Pirtskhalaishvili, R.M. (1974) Study of the transfer of amorphous boron to crystalline state. In: Tavadze, F.N., Ed., Boron Preparation, Structure, Properties, Nauka, Moscow, 23.

[3] Ugai, L.A. and Soloviev, N.F. (1977) Methods of preparations of $\alpha$-rhombohedral boron. In: Matkovich, V.I., Ed., Boron and Refractory Borides, Springer Verlag, Berlin, Heidelberg, 227-240. http://dx.doi.org/10.1007/978-3-642-66620-9_14

[4] Krutski, Ju.L., Galevski, G.V. and Kornilov, A.A. (1983) Oxidation of the ultra-disperse powders of carbide, boron, vanadium and chromium. Powder Metallurgy, 2, 47-50. (in Russian)

[5] Kervalishvili, P.D., Kutelia, E.R., Dzigrashvili, T.A., Dekanosidze, R.., and Petrov, V.I. (1985) Electron-microscopic research of the structure of the amorphous boron. Solid State Physics, 27, 1414-1418. (in Russian)

[6] Kelly, K.K. (1960) High-temperature heat-contents, heat capacity and entropy data for element and inorganic compounds. Bureau of Mines Bulletin, 584, 232 p. 